\title{
Peri-tumor fibroblasts promote tumorigenesis and metastasis of hepatocellular carcinoma via Interleukin6/STAT3 signaling pathway
}

This article was published in the following Dove Press journal: Cancer Management and Research

\section{Zhenxiong Zhao \\ Si Xiong \\ Ronghua Wang \\ Yawen Li \\ Xiju Wang \\ Yun Wang \\ Shuya Bai \\ Wei Chen \\ Yuchong Zhao \\ Bin Cheng}

Department of Gastroenterology and Hepatology, Tongji Hospital, Tongji Medical College, Huazhong University of Science and Technology, Wuhan 430030,

People's Republic of China
Correspondence: Bin Cheng Department of Gastroenterology and Hepatology, Tongji Hospital, Tongji

Medical College, Huazhong University of Science and Technology,

Jiefang Avenue 1095, Wuhan 430030,

People's Republic of China

Tel +862783663208

Fax+862783663585

Email b.cheng@tjh.tjmu.edu.cn
Purpose: Because many hepatocellular carcinoma (HCC) cases develop from fibrotic or cirrhotic livers, fibroblasts are abundant in the microenvironment of HCC. Although the contribution of cancer-associated fibroblasts (CAFs) to the progression of HCC is well established, the role of fibroblasts has not been comprehensively revealed.

Patients and methods: The RayBio Human Cytokine Antibody Array was used to elucidate the role of peri-tumor fibroblasts (PTFs) in promoting malignant properties of HCC. IL-6 and STAT3 signaling were inhibited in both HCC cell lines and non-tumor L-02 liver cells to further determine its role in the progression of HCC. Moreover, the expression of IL-6 and pTyr705 STAT3 was detected in HCC samples and peri-tumor liver tissues by immunohistochemical staining.

Results: PTFs not only promoted the proliferation, invasion, and metastasis of liver cancer cells, but also stimulated the permanent malignant transformation of human non-tumor L-02 liver cells, resulting in hepatocarcinogenesis in vivo. The RayBio Human Cytokine Antibody Array indicated that PTFs secreted a higher level of soluble IL-6 than CAFs. IL-6 derived from PTFs greatly activated STAT3 Tyr705 phosphorylation in both non-tumor L-02 cells and HCC cells. IL-6-neutralizing antibody and STAT3 Tyr705 phosphorylation inhibitor, cryptotanshinone, largely abolished the positive effects of PTFs on HCC carcinogenesis and progression. Moreover, high expression of pTyr705 STAT3 in peri-tumor tissues was significantly correlated with tumor recurrence rate after three years in a postsurgical follow-up with patients with HCC.

Conclusion: These results indicated that PTFs induce carcinogenesis and development of HCC via IL-6 and STAT3 signaling.

Keywords: PTFs, HCC, carcinogenesis, metastasis, STAT3 signaling

\section{Introduction}

Liver cancer is the fourth leading cause of cancer mortality worldwide, with about 841,000 new cases and 782,000 deaths annually. ${ }^{1}$ The most commonly recommended treatment for patients with hepatocellular carcinoma (HCC) is hepatectomy, however, over half of the patients with HCC, even those in the early stage, experience postoperative tumor recurrence within 3 years. ${ }^{2,3}$ The mechanism underlying the high rate of HCC postoperative recurrence still remains obscure.

The tumor microenvironment includes the extracellular matrix, vascular endothelial cells, immune cells, and stroma cells, which are mainly cancerassociated fibroblasts (CAFs). ${ }^{4}$ Previous reports show that CAFs contribute to 
HCC initiation and development by paracrine signaling through various kinds of inflammatory cytokines and chemokines. $^{5-7}$ For instance, CAFs stimulate malignant proliferation and tumorigenesis of $\mathrm{HCC}$ through paracrine secretion of $\mathrm{HGF}^{5}$ CAFs also secrete CCL7 and CXCL16 to enhance HCC invasion and migration. ${ }^{6}$ TGF- $\beta$ and SDF-1 derived from CAFs can decrease tumor apoptosis and promote vascular mimicry formation. ${ }^{7}$ Most studies have focused on the role of CAFs in the tumor microenvironment. However, focus should be directed toward peritumor tissues because components of the tumor microenvironment, including CAFs, are cleaned out after HCC tissue is removed from a patient's body during surgical resection. Although a recent finding discovered that peritumor fibroblasts (PTFs) strongly correlate with the development of $\mathrm{HCC}^{8}$, the mechanism concerning the role of PTFs in HCC postoperative recurrence and metastasis needs to be further explored.

In the present study, we showed that PTFs have a strong effect on stimulating the transformation of nontumor L-02 liver cells into malignant cells and enhancing tumor proliferation, invasion and metastasis. Subsequently, PTF-derived and IL-6-mediated STAT3 Tyr705 phosphorylation plays a critical role in this process. These findings suggest that PTF targeting should be considered during surgical resection and could be an effective therapeutic strategy against the postoperative recurrence and metastasis of HCC.

\section{Material and methods}

\section{Liver tissue specimen of HCC patients}

Human liver tumor and peri-tumor tissues were obtained from $88 \mathrm{HCC}$ patients who underwent surgical resection in Tongji Hospital, Huazhong University of Science and Technology (Wuhan, China). Peri-tumor tissues were sampled $1 \mathrm{~cm}$ away from the border of the cancer mass. Clinical data associated with these specimens were also recorded. All human experimentations were approved by the ethics committee of Tongji Hospital. The patients whose tissues were used in this research provided written informed consent, and this was conducted in accordance with the Declaration of Helsinki.

\section{Immunohistochemistry analysis}

Immunohistochemical staining was performed on formalin-fixed, paraffin-embedded liver tissue sections by labelled streptavidin biotin peroxidase complex method.
For immunohistochemical analysis, staining for IL-6 and p-STAT3(Tyr705) was carried out, using rabbit polyclonal antibodies against IL-6(cat\#ab9324; Abcam) and p-STAT3 (Tyr705) (cat\#9145; Cell Signaling Technology) both at a dilution of 1:200. The intensity of staining was scored on a four-point scale as negative (0), weak (1), moderate (2), or strong (3). The percentage of positively stained tissue area relative to the entire tissue area was scored on a scale of $0-4,0(0 \%), 1(1-25 \%), 2$ (26-50\%), 3 (51-75\%), and $4(76-100 \%)$ respectively. An overall protein expression score (range 0-12) was calculated by multiplying the staining intensity and positive staining scores. Scores $<6$ were regarded as low expression, while scores $\geq 6$ were considered as high expression. The scores from three observers were collected to calculate the mean value of the final score.

\section{Isolation and purification of CAFs, PTFs and NFs}

Paired peri-tumor and tumor tissues from five patients were harvested and freshly processed, and peri-tumor tissues were sampled $1 \mathrm{~cm}$ far away from tumor tissues. These patients did not undergo chemotherapy before surgery. CAFs and PTFs were isolated from tumor and peritumor tissues, while normal fibroblasts (NFs) were isolated from liver tissues adjacent to hepatic hemangioma. The fresh liver tissues were sliced into $2-3 \mathrm{~mm}$ fragments, washed in D-Hanks solution containing $100 \mathrm{U} / \mathrm{mL}$ penicillin and $100 \mathrm{ug} / \mathrm{mL}$ streptomycin, and then plated in a culture dish with DMEM with 15\% fetal bovine serum. Fibroblasts were observed to grow out of tumor fragments for $1-2$ weeks at $37{ }^{\circ} \mathrm{C}$ and $5 \% \mathrm{CO}$. We obtained $95 \%$ purified fibroblasts after 2-3 passages. CAFs, NFs and PTFs from passages 3-10 were later used for various experiments.

\section{Immunofluorescence}

CAFs, PTFs and NFs, were tested for the expression of vimentin, fibroblast activation protein (FAP), and $\alpha$-SMA by immunofluorescence at passage 4 . Cells were seeded on coverslips in 24 -well chamber slides, fixed with $4 \%$ paraformaldehyde for $20 \mathrm{~min}$ and permeabilized with $0.1 \%$ Triton X-100 for $10 \mathrm{~min}$. After blocking with 10\% bovine serum albumin (BSA), the cells were incubated with primary antibodies against anti-alpha-smooth muscle actin ( $\alpha$-SMA) (Boster, dilution 1:100), FAP (Boster, dilution $1: 100$ ) and Vimentin (Boster, dilution 1:100) at $4{ }^{\circ} \mathrm{C}$ 
overnight, followed by staining with a fluorescein isothiocyanate (FITC)-labeled goat anti-rabbit or goat anti-mouse secondary antibody $(1: 1,000)$ for $1 \mathrm{~h}$ at $37^{\circ} \mathrm{C}$ in a dark room. Then DAPI staining (4',6-diamidino-2- phenylindole) was performed for 30 mins for visualizing nuclei. The coverslips were blocked with Antifade Mounting Medium.

\section{Cell lines}

The human non-tumor hepatic cell line L-02 cells was generously provided by Dr. Xinyuan Liu (Shanghai Institutes for Biological Sciences, Shanghai, China) and the use of L-02 cells was approved by the Ethics Committee of Tongji Hospital. Human HCC cell lines (MHCC-97H and Huh-7) were obtained from the Cell Bank of the Chinese Academy of Sciences (Shanghai, China). All cell lines were cultured in Dulbecco's modified Eagle's medium (DMEM; GIBCO, Grand Island, NY, USA) containing 10\% fetal bovine serum (FBS; GIBCO). Cells were incubated at $37{ }^{\circ} \mathrm{C}$ with $5 \% \mathrm{CO} 2$ environment.

\section{Reagents and antibodies}

Cryptotanshinone $(10 \mu \mathrm{M})$ was purchased from MedChemExpress and Western blot antibodies for STAT3 (cat\#8768), and p-STAT3 (cat\#9145) were purchased from Cell Signaling Technology, Inc. (Danvers, MA, USA), 1:2,000 dilution. Monoclonal mouse anti- $\beta$-actin (cat\#ab8226; Abcam, 1:1,000 dilution) was used as an internal control.

\section{Lentiviral-based knockdown of STAT3 pathway}

To suppress the STAT3 signaling pathway in MHCC-97H cells, lentiviral particles (Genechem, Shanghai) expressing STAT3-siRNA were used in our study. The siRNA sequence targeting STAT3 was listed as follows: 5'CGGCAACAGATTGCCTGCATT-3', while negative control was TTCTCCGAACGTGTCACGT. The cells infected were selected for further experiments.

\section{Xenograft tumor model}

Male BALB/c- nude mice (weighing approximately $19 \mathrm{~g}$; aged 4 weeks) were purchased from Beijing Huafukang Bioscience and maintained in pathogen-free conditions. All animal experiments were approved by the Institutional Animal Care and Use Committee of the Huazhong University of Science and Technology. Guidelines for Experimental Animal Ethical Committee of Huazhong University of Science and
Technology and Experimental animals administrative regulations of Hubei Province were followed for the welfare of the animals. Each treatment group consisted of 3 random mice who were housed in the animal centre of Tongji Medical College, Huazhong University of Science and Technology (Wuhan, China). We resuspended MHCC-97H cells and L-02 cells alone or together with PTFs in serum-free medium, combining together with matrigel in a 1:1 ratio and injected them subcutaneously into flanks of the nude mice. The length (L) and width (W) of the tumors were measured externally every 2 days as from the seventh day. Tumor volume was calculated with the formula: $\mathrm{V}=(\mathrm{L} \times \mathrm{W} 2) / 2$. After 14 days, all mice were sacrificed, and the tumors were excised and measured. AFP Antibody (cat\#14550-1, proteintech) was used for Immunohistochemical staining of the tumors at a dilution of $1: 50$.

\section{Colony formation}

To determine cellular ability for proliferation, a density of 1,000 cells $/ \mathrm{mL}$ were placed in 6 well plates and medium with different treatment was used to culture cells for 1 week. Colonies were fixed with methanol and stained with methylene blue.

\section{Cell migration and invasion assay}

Transwell chambers without Matrigel were used to examine cell ability of migration and transwell chambers were coated with 50 ul Matrigel to measure invasion. $5 \times 104$ cells were serum-starved for $12 \mathrm{~h}$ and resuspended in $100 \mu \mathrm{L}$ serum-freed medium and were added to the upper compartment of the chamber, while the bottom chamber was filled with medium supplemented with $10 \%$ FBS. After incubation at $37{ }^{\circ} \mathrm{C}$ in a $5 \% \mathrm{CO} 2$ humidified atmosphere, the chambers were analyzed $24 \mathrm{hrs}$ later for migration and $48 \mathrm{hrs}$ for invasion. The assay was repeated at least three times.

\section{Preparation of conditioned medium (CM)}

Following co-culture with HCC cells for $48 \mathrm{hrs,} \mathrm{CAFs,} \mathrm{NFs}$ and PTFs were plated in serum-free DMEM for $36 \mathrm{hrs}$. Then, the medium was collected from the supernatants after centrifugation and filtration through a $0.45 \mathrm{~mm}$ membrane to remove any cells and cell debris. Conditioned medium obtained were stored at $-80{ }^{\circ} \mathrm{C}$.

\section{Statistical analysis}

Statistical analysis was performed using SPSS (v20.0; SPSS, Inc., Chicago, IL, USA) software. Chi-square ( $\chi 2)$ 
tests and Fisher's exact tests (for nominal variables) were used for analyzing clinical and pathological parameters. Two-group comparisons was performed with unpaired Student's $t$-test. The data were presented as mean \pm standard deviation. Statistical significance was defined as $P<0.05$.

\section{Results}

\section{Isolation and identification of CAFs, NFs and PTFs}

Fibroblasts were isolated and cultivated by primary culture methods. Approximately two weeks after small pieces of HCC tumors, peri-tumor tissues, and normal liver tissues were put into culture dishes, fibroblasts gradually migrated outside of the tissues (Figure S1A). The three types of fibroblasts observed were morphologically similar (Figure S1B). Immunofluorescence was used to examine mesenchymal markers, including $\alpha$-smooth muscle actin ( $\alpha$-SMA), Vimentin, and fibroblast activation protein (FAP), in isolated fibroblasts. CAFs exhibited the highest expression level of these three markers, while the expression of FAP was lower in NFs and almost nonexistent in PTFs. And $\alpha$-SMA expression was higher in PTFs than NFs (Figure S1C-E). None of the fibroblasts expressed endothelial marker CD31 or macrophage marker CD68 (Figure S2).

We previously performed immunohistochemical staining to locate CAFs and PTFs in tumor and peri-tumor tissues. We have also confirmed that the expression of $\alpha$ SMA in tumors was significantly higher than in adjacent non-tumor tissues. ${ }^{9}$

\section{PTFs promote the proliferation, invasion, and metastasis of $\mathrm{HCC}$ cells}

After co-culture with MHCC-97H cells for $48 \mathrm{~h}$, stimulated CAFs, NFs, and PTFs were removed and cultivated alone. Fibroblast supernatants were collected individually as CAFs-CM, NFs-CM and PTFs-CM to culture HCC cells. Colony formation assays determined whether PTFs would affect the proliferation of HCC cells and revealed that all the conditioned mediums from the three different fibroblasts enhanced HCC cell proliferation. Surprisingly, PTFs-CM stimulated the proliferation stronger than CAFs$\mathrm{CM}$ (Figure 1A). Additionally, Matrigel invasion and transwell migration assays indicated that PTFs-CM greatly stimulated the invasion and migration of HCC cells, which was also stronger than the stimulation by CAFs-CM (Figure $1 \mathrm{~B}$ and $\mathrm{C}$ ). These findings highlight the role of PTFs in enhancing the progression and metastasis of HCC.

\section{IL-6 secreted from PTFs accelerate the development of HCC}

To uncover the mechanism of PTFs' effect on HCC development, we used the RayBio Human Cytokine Antibody Array to analyze CAFs-CM, PTFs-CM, and the supernatant of MHCC-97H cells. We have previously demonstrated that MHCC-97H cells secrete much less IL-6 than CAFs, and IL-6 is up-regulated most in CAFs-CM compared to HCC cells. ${ }^{9}$ Here, our array found that the gray value of soluble IL-6 was higher in PTFs-CM than in CAFs-CM (Figure 2A). This was also confirmed by an Elisa assay (Figure 2B), which showed that PTFs secreted higher amounts of IL-6 than CAFs. The level of IL-6 produced by MHCC-97H cells was much lower, when compared to CAFs or PTFs.

As shown in Figure 2C, IL-6 added into DMEM largely increased the migration and invasion of HCC cells in vitro when compared to controls. Furthermore, incubation with IL-6-neutralizing antibody in PTFs-CM mostly abolished the effect of PTFs on enhancing HCC invasion and migration (Figure 2D). Conclusively, IL-6 derived from PTFs played a major role in accelerating the progression and metastasis of HCC.

\section{PTFs-derived IL-6 promotes HCC progression through activation of STAT3 Tyr705 phosphorylation both in vitro and in vivo}

Previous studies revealed that IL-6 was mostly correlated with the STAT3 signaling pathway, contributing to HCC development. $^{9-11}$ To investigate this hypothesis in terms of PTFs' effects on HCC, we detected the activation of STAT3 signaling in MHCC-97H cells cultured with DMEM, NFs$\mathrm{CM}$, CAFs-CM, and PTFs-CM, respectively. This demonstrated that the level of STAT3 Tyr705 phosphorylation in HCC cells increased greatly when cultured in PTFs-CM compared to the control groups (Figure 3A). IL-6 alone also effectively stimulated the activation of STAT3 signaling in MHCC-97H cells. The maximum effect was observed within thirty minutes after incubation with IL-6 added to DMEM (Figure 3B). Moreover, the effect of PTFs-CM was mostly reduced when IL-6 was removed by the IL-6-neutralizing antibody (Figure 3C).

To determine the role of the STAT3 pathway in HCC progression, we treated MHCC-97H cells with cryptotanshinone (CTS), which is an inhibitor of STAT3 Tyr705 phosphorylation. CTS effectively abolished STAT3 Tyr705 
A
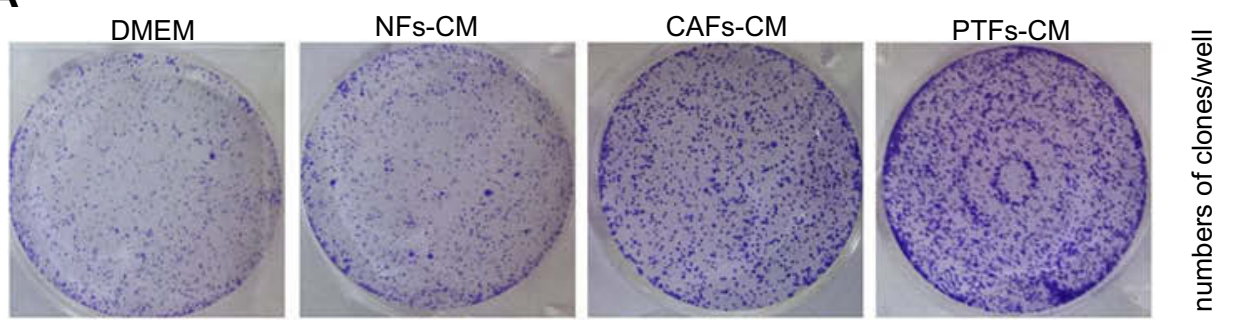

B

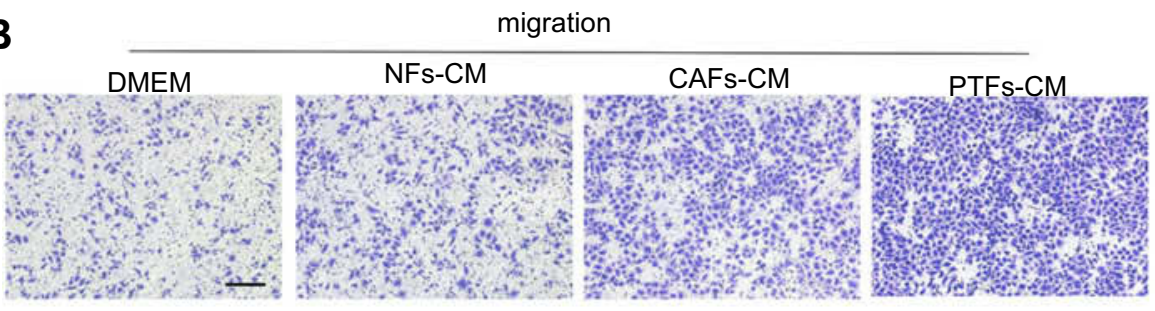

C

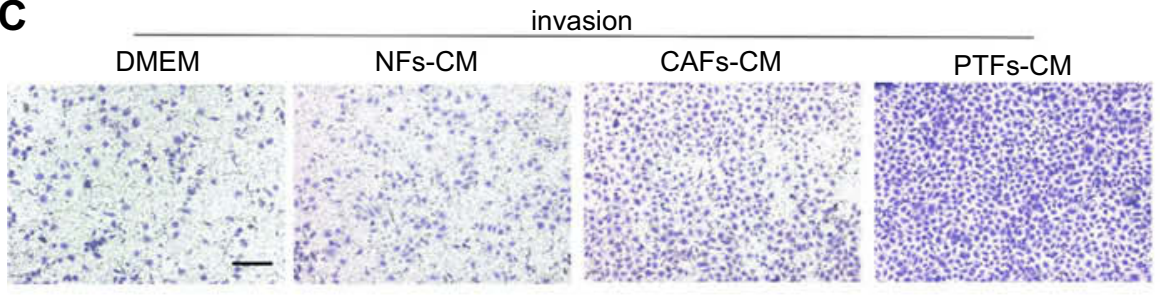
nvasion
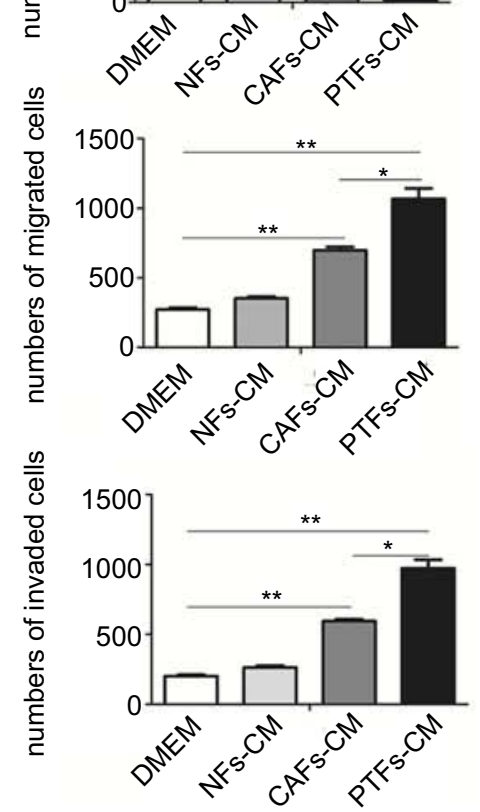

Figure I PTFs promoted the proliferation, invasion and migration of $\mathrm{HCC}$ cells in vitro

Notes: (A-C) MHCC-97H cells were incubated with DMEM, NFs-CM, CAFs-CM and PTFs-CM for 48 h respectively. Then the cells were subjected to colony-formation assay $(\mathbf{A})$ and transwell assay $(\mathbf{B}, \mathbf{C})$. The numbers of cells that formed colons, invaded and migrated were calculated and analyzed. Scale bar, $200 \mu \mathrm{m}$. Data are shown as mean \pm SD from at least three independent experiments $(* P<0.05, * * P<0.01)$.

Abbreviations: CM, conditioned medium; HCC, hepatocellular carcinoma; CAFs, cancer-associated fibroblasts; HCC, hepatocellular carcinoma; NFs, normal fibroblasts; PTFs, peri-tumor fibroblasts.

phosphorylation in HCC cells, even when cells were cultured in PTFs-CM or IL-6-added DMEM (Figure 3D). Inhibition of pTyr705 STAT3 in MHCC-97H cells mainly blocked the effect of IL-6- and PTFs-CM-induced migration and invasion of the cells (Figure 3E and F). Consistently, PTFs could also promote the invasion and migration of Huh 7 cells via IL6 and STAT3 signaling pathway (Figure S3).

To further verify whether PTFs promote HCC growth via activation of STAT3 signaling in vivo, we transfected MHCC-97H cells with lentiviral plasmids containing STAT3-shRNA (shSTAT3) or a vector control (shCtl) and then subcutaneously injected the cells into the flanks of BALB/c nude mice together with PTFs. ShCtl MHCC-97H cells and PTFs were also injected alone. Injection with MHCC-97H cells and PTFs resulted in much larger tumors than MHCC-97H cells alone, and this effect was greatly reduced by STAT3 knockdown. Injection with PTFs alone did not result in tumor development (Figure 3G).

\section{High pTyr705 STAT3 expression in peri-tumor tissue correlates with postsurgical tumor recurrence in HCC patients}

We evaluated the expression of IL-6 and pTyr705 STAT3 in 88 pairs of HCC samples and matched peritumor tissue by immunohistochemical staining. Both IL6 and pTyr705 STAT3 expression were upregulated in peri-tumor tissue relative to tumor tissue (Figure 4). Correlations between the expression of pTyr705 STAT3 and clinical-pathological features are presented in Table 1. High expression of p-STAT3 in peri-tumor tissues was significantly associated with poor cancer differentiation $(P=0.028)$ and postsurgical tumor recurrence after three years $(P=0.034)$, in patients with HCC. Therefore, high p-STAT3 expression in peri-tumor tissues indicated a poor prognosis for patients with HCC. 
A
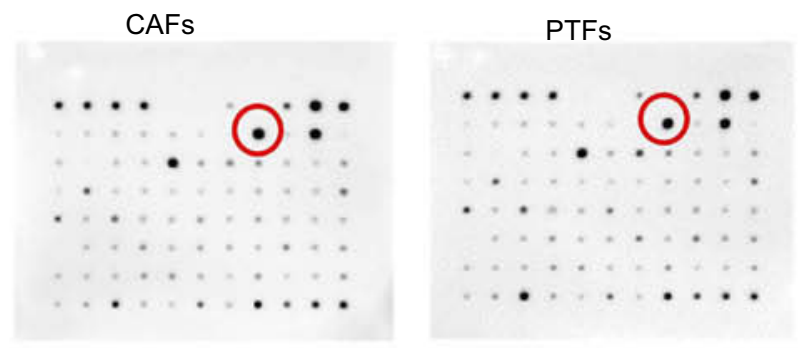

B

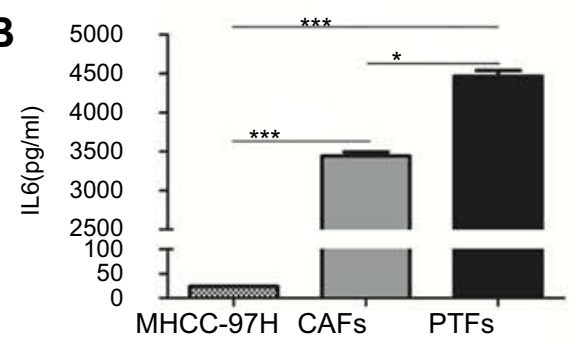

C

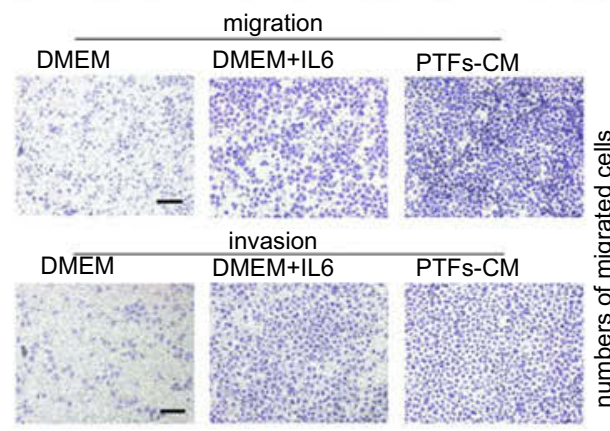

D
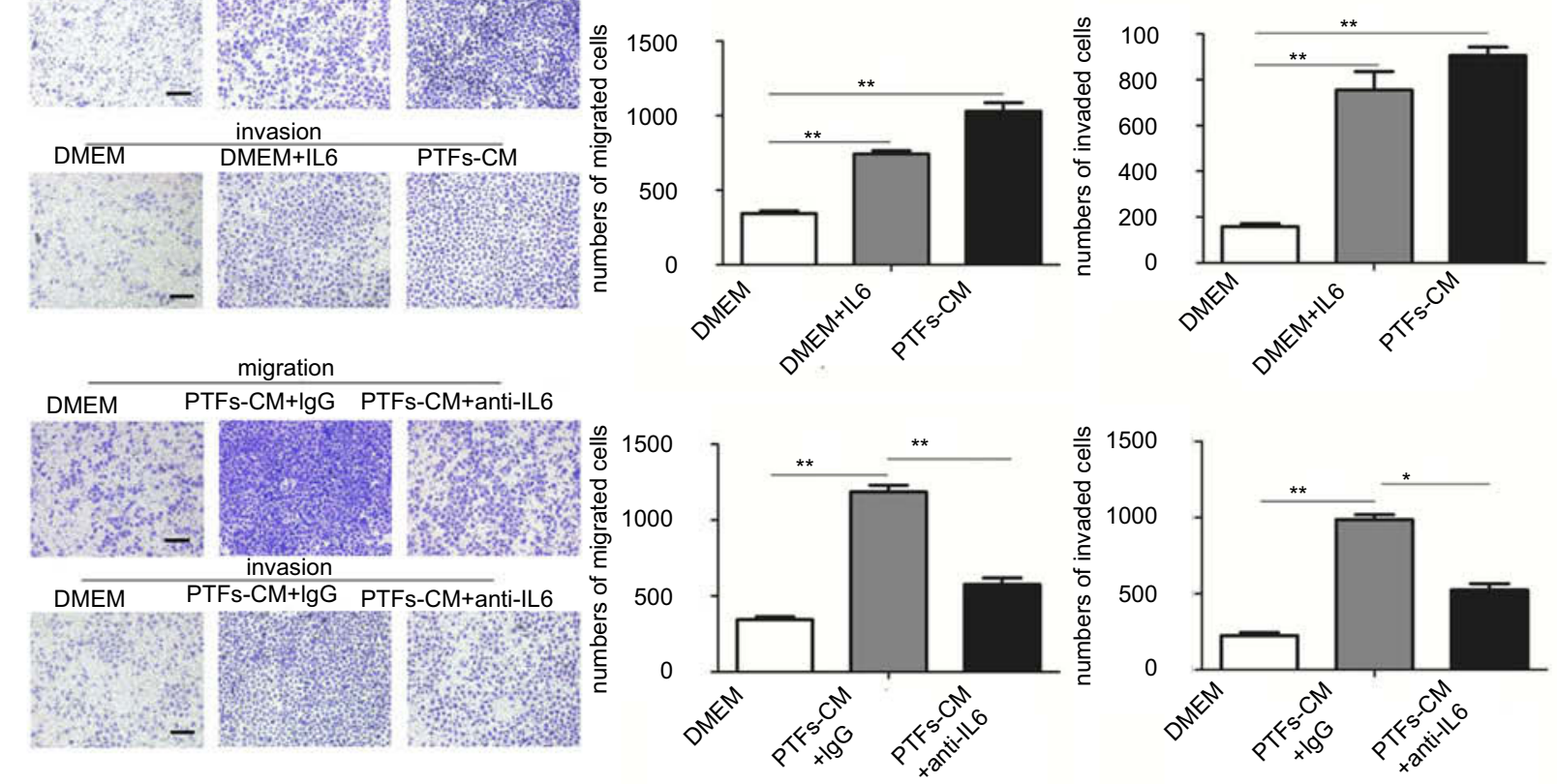

Figure 2 PTFs promoted the progression of HCC mainly by secreting Interleukin 6.

Notes: (A) The profiles of the cytokines secreted from CAFs and PTFs was examined by using the RayBio Human Cytokine Antibody Array. The most significantly increased chemokine IL-6 was marked. (B) Elisa assay was performed to detect the amount of soluble IL-6 in each group. (C) MHCC-97H cells cultured with DMEM containing soluble IL-6 $(40 \mathrm{ng} / \mathrm{ml})$ were assessed by transwell assay. (D) IL-6 neutralizing antibody decreased the effect of PTFs-CM on the migration and invasion of MHCC$97 \mathrm{H}$ cells. Scale bar, $200 \mu \mathrm{m}$. Data are shown as mean \pm SD from at least three independent experiments $(* P<0.05, * * P<0.0 \mathrm{I}, * * * P<0.00 \mathrm{I})$.

Abbreviations: CAFs, cancer-associated fibroblasts; CM, conditioned medium; HCC, hepatocellular carcinoma; PTFs, peri-tumor fibroblasts.

\section{Non-tumor L-02 cells were transformed into malignant cells by PTFs}

To further determine the role of PTFs on hepatocarcinogenesis, we first grew human non-tumor L-02 liver cells in PTFs-CM or DMEM for $48 \mathrm{~h}$. Then, tumorigenic indicator changes (proliferation and migration) in L-02 cells were measured. Our data from colony formation assays indicated that PTFs-CM stimulated faster proliferation of L-02 cells when compared to DMEM (Figure 5A). Transwell assays showed substantially more migrating L-02 cells when co-cultured with PTFs, compared to the control group, indicating that PTFs promote migration of L-02 cells (Figure 5B). Thus, we concluded that PTFs stimulate the malignant phenotype in L-02 cells in vitro.
Next, we cultivated L-02 cells with PTFs-CM to further determine whether PTFs influence L-02 cells by IL-6 and p-STAT3 signaling. A strong effect of STAT3 phosphorylation in hepatocytes was observed by western blot. Both the IL-6-neutralizing antibody and CTS were able to block activation of the STAT3 pathway by PTFs in L-02 cells (Figure 5C). IL-6 alone also activate the STAT3 pathway in L-02 cells, and the effect was maximized at thirty minutes after incubation (Figure 5D). As shown in Figure 5E and F, both the IL-6-neutralizing antibody and inhibiting pTyr705 STAT3 abolished the enhancing effects of PTFs on L-02 cell proliferation and migration, thus preventing non-tumor L-02 liver cells from turning malignant. 
A

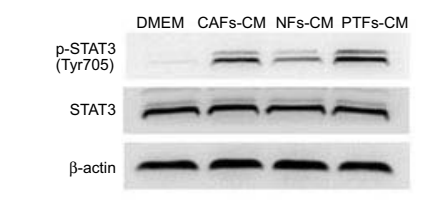

B
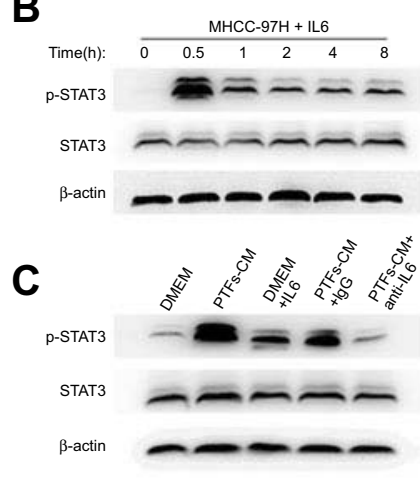

D

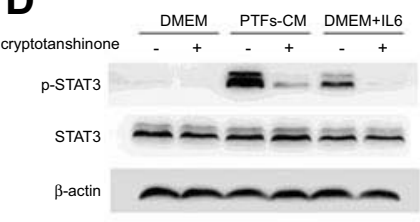

E

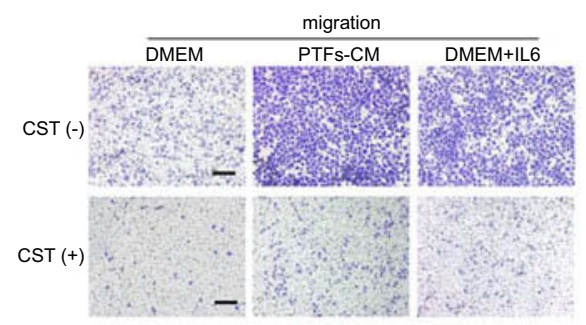

F
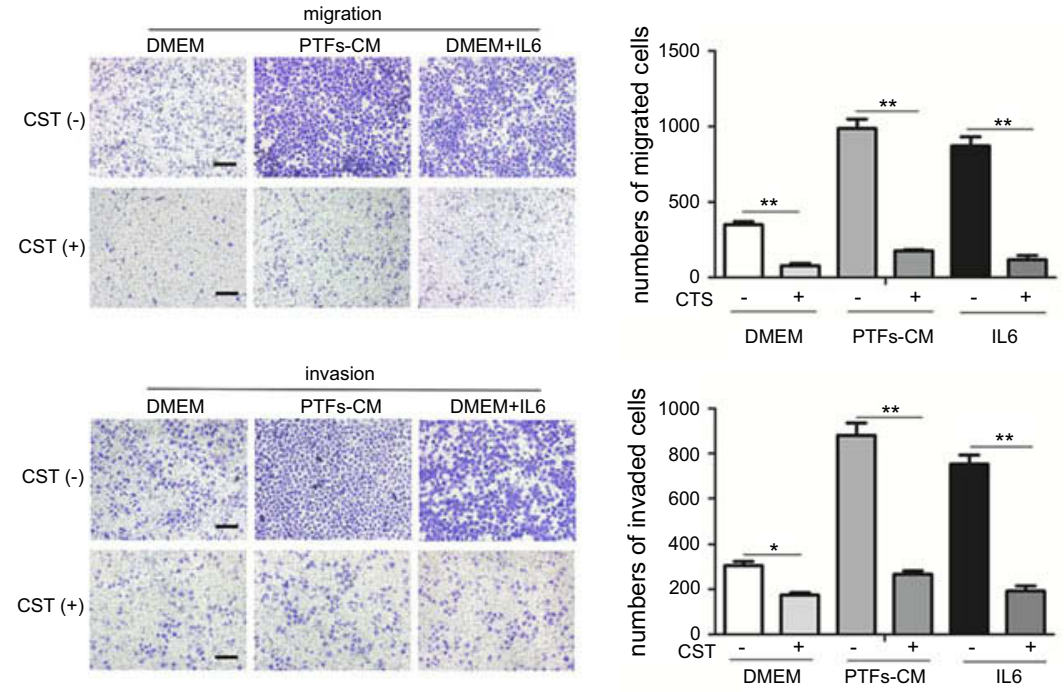

G
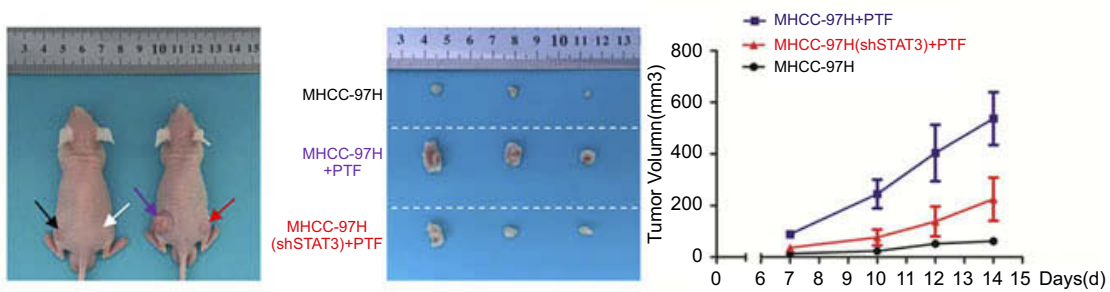

Figure 3 PTFs-derived IL-6 induced the invasion and metastasis of HCC cells through activation of STAT3 signaling.

Notes: (A) MHCC-97H cells were treated with DMEM, NFs-CM, CAFs-CM and PTFs-CM for $48 \mathrm{~h}$ respectively. The phosphorylation of STAT3 was detected by Western blot. (B) MHCC-97H cells were incubated with IL-6 $(40 \mathrm{ng} / \mathrm{ml})$. (C) IL-6 neutralizing antibody abolished the effect of PTFs on promoting STAT3 phosphorylation (Tyr705) in MHCC-97H cells. (D) MHCC-97H cells cultured in DMEM, PTFs-CM and IL-6 added DMEM were treated with cryptotanshinone, an STAT3 Tyr705 phosphorylation inhibitor, for 24 h. (E+F) Both IL-6- and PTFs-induced migration (E) and invasion (F) abilities of MHCC-97H cells were reduced by cryptotanshinone as assessed by transwell assays. Scale bar $200 \mu \mathrm{m}$. Data are shown as mean \pm SD from at least three independent experiments $(* P<0.05$, $* * P<0.01)$. (G) MHCC-97H cells transfected with lentiviral plasmid containing STAT3-shRNA (shSTAT3) or vector control (shCtl) were subcutaneously injected into flanks of BALB/c nude mice together with PTFs. MHCC97H(shCtl) and PTFs were also injected alone. Tumors formed at each group were shown $n=3$. Black arrow means just injecting MHCC-97H cells, white arrow means PTFs, purple arrow means injecting MHCC-97H(shCtl) with PTFs and red arrow means MHCC-97H(shSTAT3) together with PTFs.

Abbreviations: CM, conditioned medium; HCC, hepatocellular carcinoma; CAFs, cancer-associated fibroblasts; HCC, hepatocellular carcinoma; NFs, normal fibroblasts; PTFs, peri-tumor fibroblasts.

Eventually, to determine the role of PTFs on the malignant transformation of non-tumor L-02 cells in vivo, $10^{4}$ L-02 cells were subcutaneously inoculated alone or together with PTFs into the flanks of BALB/c nude mice (Figure 6A). Two weeks later, mice models injected with L-02 cells or PTFs alone did not develop tumors. However, tumors were detected when L-02 cells were combined with PTFs and were inoculated into the skin of the nude mice. Pathological examination by hematoxylin and eosin staining of the primary tumors confirmed malignant phenotypes and IHC of the tumor showed positive expression of HCC special marker AFP (Figure S4). From those data, we established that PTFs stimulate the permanent malignant transformation of non-tumor hepatocytes and contribute to the carcinogenesis of HCC in vivo. Furthermore, L-02 cells, with pTyr705 STAT3 inhibited by CTS were also injected subcutaneously along with
PTFs into BALB/c nude mice, forming much smaller tumors than the control group, with one in three mice exhibiting no tumors (Figure 6B). Consistently, PTFs could promote tumor progression of $10^{5} \mathrm{~L}-02$ cells via STAT3 activation (Figure S5). Based on these findings, we hypothesized that STAT3 signaling activation might be required for PTFs to promote HCC tumorigenesis.

In conclusion, we postulated that PTFs produce large amounts of IL-6, which continuously activates STAT3 phosphorylation and results in a malignant phenotype in non-tumor L-02 liver cells, promoting carcinogenesis and progression of HCC.

\section{Discussion}

The idea that the tumor microenvironment contributes to cancer initiation and development is widely believed. ${ }^{12-14}$ However, when tumor tissue is removed 


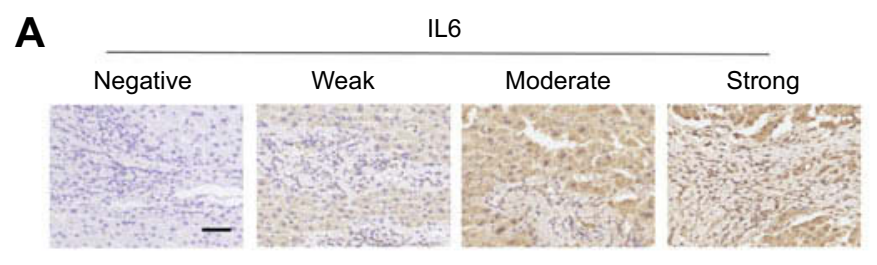

B

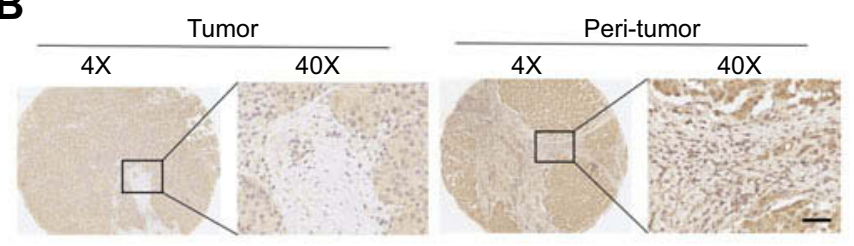

C

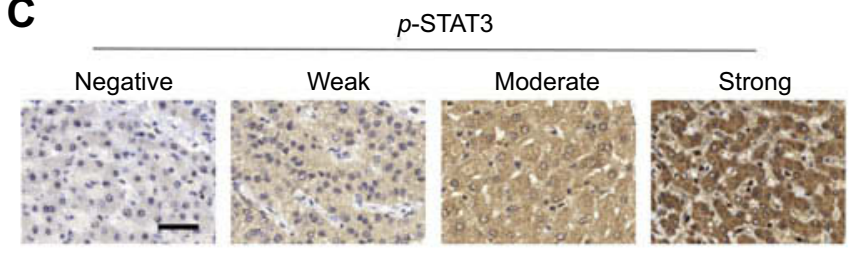

D

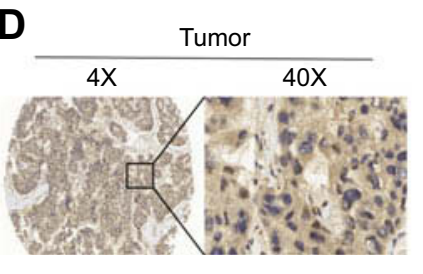

E

IL6

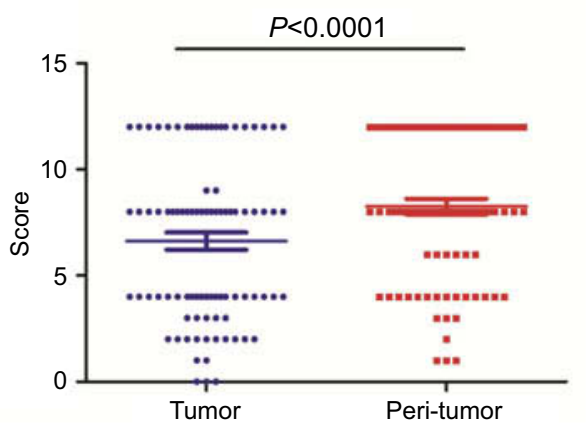

F

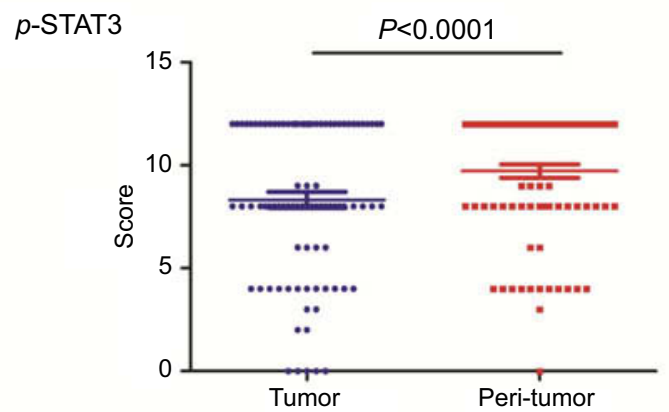

Figure 4 Both the levels of IL-6 and P-STAT3(Tyr705) expression are higher in peri-tumor tissues than tumor tissues.

Notes: $(\mathbf{A}+\mathbf{C})$ The photograph shows representative tissues with four different levels (negative, weak, moderate, or strong) of immunohistochemical staining intensity of IL6 and p-STAT3 (Tyr705). Scale bar, $50 \mu \mathrm{m}$. (B+D) The expression levels of IL-6 and pTyr705 STAT3 in peri-tumor tissues were higher than those in tumor tissues by immunohistochemistry. (E+F) Analysis of the expression levels of IL-6 and p-STAT3 (Tyr705) in 88 pairs of tumor and adjacent non-tumor tissues by paired t-test.

from patients with HCCs' livers by surgical resection, the tumor cells and their microenvironment have been completely cleared. Unfortunately, PTFs remain inside the liver with normal hepatocytes in adjacent non-tumor tissues (Figure S6).

A previous study reported that high M-CSF expression in the peritumoral liver tissue, but not in the tumor tissue, is significantly associated with a high incidence of intrahepatic metastasis and poor survival after resection of HCC. ${ }^{15}$ In patients receiving curative HCC resection, a high density of peritumoral activated hepatic stellate cells infiltration correlates with a 2.6-fold hazard ratio for overall survival and a 3.3-fold hazard ratio for recurrencefree survival. ${ }^{16}$ Furthermore, a recent study demonstrated that PTFs might be involved in HCC development by secreting various cytokines. ${ }^{8}$ However, the underlying mechanism of PTFs' effect on HCC remains unclear and the role of PTFs in postoperative tumor relapse requires further investigation.
Our study demonstrates that PTFs stimulate the malignant transformation of non-tumor liver cells and induce carcinogenesis and metastasis of HCC via the IL-6 and STAT3 signaling pathway. High expression of pTyr705 STAT3 in peri-tumor tissue strongly correlated with tumor recurrence rate three years after surgery for patients with HCC. Therefore, we postulate that PTFs contribute to the postoperative relapse and metastasis of HCC.

The primary focus of oncology should not be epithelial cells or, more specifically, the genetic changes and oncogenic signaling pathways of epithelial cells as they progress from normal to malignant. The environment also contributes substantially to the pathology and may promote the accumulation of oncogene activation in epithelial cells, thereby inducing tumorigenesis and cancer development. ${ }^{17-19}$ Cunha et al showed that CAFs contributed to the tumor progression of initiated human prostatic epithelial cells in vivo. Furthermore, non-tumor human prostatic epithelial cells may be permanently transformed by CAFs and become tumorigenic..$^{20,21}$ 
Table I Correlation between p-STAT3 expression in HCC peri-tumor issues and clinicopathological parameters $(n=88)$

\begin{tabular}{|c|c|c|c|}
\hline $\begin{array}{l}\text { Clinicopathological } \\
\text { features }\end{array}$ & $\begin{array}{l}\text { High p- } \\
\text { STAT3 } \\
(n=75)\end{array}$ & $\begin{array}{l}\text { Low p- } \\
\text { STAT3 } \\
(n=13)\end{array}$ & $\begin{array}{l}P \\
\text {-value* }\end{array}$ \\
\hline $\begin{array}{l}\text { Age } \\
\qquad \begin{array}{l}<40 \text { years } \\
\geq 40 \text { years }\end{array}\end{array}$ & $\begin{array}{l}58 \\
17\end{array}$ & $\begin{array}{l}10 \\
3\end{array}$ & I \\
\hline $\begin{array}{l}\text { Gender } \\
\text { Male } \\
\text { Female }\end{array}$ & $\begin{array}{l}65 \\
10\end{array}$ & $\begin{array}{l}13 \\
0\end{array}$ & 0.355 \\
\hline $\begin{array}{l}\text { Hepatitis B } \\
\text { Negative } \\
\text { Positive }\end{array}$ & $\begin{array}{l}14 \\
61\end{array}$ & $\begin{array}{l}2 \\
11\end{array}$ & I \\
\hline $\begin{array}{l}\text { Tumor size } \\
\quad<6 \mathrm{~cm} \\
\geq 6 \mathrm{~cm}\end{array}$ & $\begin{array}{l}24 \\
51\end{array}$ & $\begin{array}{l}5 \\
8\end{array}$ & 0.890 \\
\hline $\begin{array}{l}\text { Histological } \\
\text { differentiation* } \\
\text { Moderate/Well } \\
\text { Poor }\end{array}$ & $\begin{array}{l}33 \\
42\end{array}$ & $\begin{array}{l}10 \\
3\end{array}$ & $0.028^{*}$ \\
\hline $\begin{array}{l}\alpha \text {-fetoprotein level } \\
\quad<400 \mathrm{ng} / \mathrm{mL} \\
\quad \geq 400 \mathrm{ng} / \mathrm{mL}\end{array}$ & $\begin{array}{l}38 \\
37\end{array}$ & $\begin{array}{l}7 \\
6\end{array}$ & 0.832 \\
\hline $\begin{array}{l}\text { Postsurgical recur- } \\
\text { rence in } 3 \text { years * } \\
\text { Absence } \\
\text { Presence }\end{array}$ & $\begin{array}{l}16 \\
59\end{array}$ & $\begin{array}{l}7 \\
6\end{array}$ & $0.034 *$ \\
\hline $\begin{array}{l}\text { Lymph node } \\
\text { metastasis } \\
\text { Absence } \\
\text { Presence }\end{array}$ & $\begin{array}{l}61 \\
14\end{array}$ & $\begin{array}{l}12 \\
1\end{array}$ & 0.567 \\
\hline $\begin{array}{l}\text { Venous Infiltration } \\
\text { Absence } \\
\text { Presence }\end{array}$ & $\begin{array}{l}61 \\
14\end{array}$ & $\begin{array}{l}11 \\
2\end{array}$ & I \\
\hline
\end{tabular}

Note: $* P<0.05$, Significant difference $(\chi 2$ test and Fisher's exact test).

Abbreviations: HCC, hepatocellular carcinoma; IL-6, interleukin 6; STAT3, signal transducer and activator of transcription 3; CAFs, cancer-associated fibroblasts: PTFs, peri-tumor fibroblasts; NFs, normal fibroblasts; CM, conditioned medium; $\alpha$ SMA, alpha-smooth muscle actin; FAP, fibroblast activation protein.

Most cases of HCC arise in the presence of advanced fibrosis or cirrhosis. The tumor stroma is essentially the supporting architecture of HCC initiation and progression. As a major component of the stroma, the effects of CAFs on promoting HCC tumorigenesis and progression are extensively defined. ${ }^{6,22,23}$ However, fewer studies have focused on PTFs. We have demonstrated that PTFs contribute greatly to the carcinogenesis and tumor growth of HCC. Non-tumor liver cells injected alone into BALB/c nude mice subcutaneously did not cause tumors, but malignant tumors developed when the liver cells were injected together with PTFs.

Soluble mediators secreted by the stroma directly affect the malignant hepatocytes, which results in altered oncogenic pathways during cancer progression. As a major inflammatory mediator, IL-6 plays an important role in the progression of $\mathrm{HCC}$ and various other cancers. ${ }^{24-26}$ In our study, IL-6 levels were significantly higher in PTFs-CM than CAFs-CM. PTFs' ability to stimulate HCC tumorigenesis and development mainly relied on secreted IL-6. The ability was almost abolished with the addition of IL6-neutralizing antibody. STAT3 activation is observed in many cancers, typically acting as a critical oncogenic mediator and inducing malignancy. ${ }^{27,28}$ In hepatitis B virus-related HCC, blocking STAT3 signaling largely inhibits tumor growth. ${ }^{29}$

The link between IL-6 and p-STAT3 has been reported in various cancers. ${ }^{9-11,30,31}$ In breast cancer, IL-6 and STAT3 signaling mediates the crosstalk between MDSCs and $\mathrm{CSCs}^{30}$ and promotes tumor progression in gastric cancer. ${ }^{31}$ IL-6 and STAT3 signaling plays a critical role in the carcinogenesis and development of HCC. ${ }^{9-11}$

IL-6 can activates STAT3 transiently. However, the mechanistic reason underlying the persistent activation of STAT3 signaling in cancer cells is not clear. Our results suggest that IL-6, which is the most highly secreted cytokine from PTFs, might be the leading precursor for STAT3 activation. This is true not only in non-tumor L-02 liver cells during tumorigenesis, but also in HCC cells during cancer progression. IL-6 alone stimulates transient STAT3 Tyr705 phosphorylation, while PTFs induce long-lasting STAT3 activation by continuously secreting IL-6. Blockade of the IL-6 and STAT3 signaling pathway by anti-human IL-6-neutralizing antibodies and pTyr705 STAT3- specific inhibitors significantly suppressed PTFsCM-induced HCC malignancy. We also confirmed that the expression of IL-6 and p-STAT3 is upregulated more in peri-tumor tissues than in tumor areas. Moreover, high p-STAT3 expression in adjacent non-tumor tissues correlated with poor postoperative outcomes for patients with HCC. Our data is consistent with clinical findings that high serum IL-6 is a risk factor and strongly associated with poor prognosis for patients with HCC. ${ }^{32,33}$

Our study does not exclude other potential mechanisms by which PTFs may promote tumorigenesis and progression of HCC. There are other cytokines that can promote the activation of STAT3 signaling. For instance, IL-11 and 
A
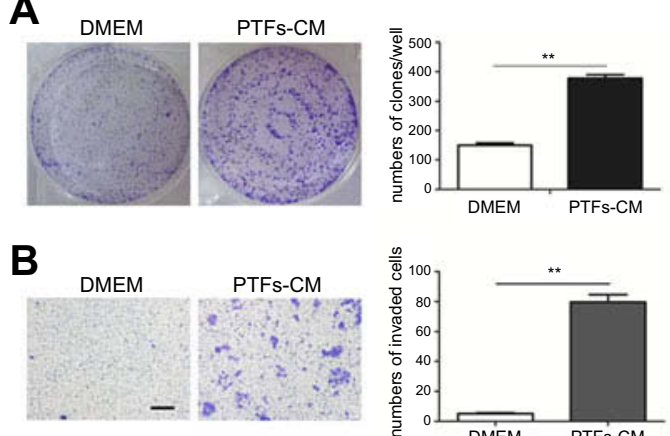

E
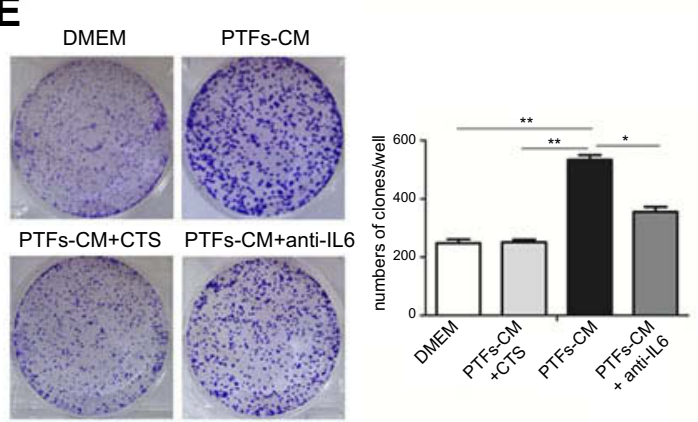

C

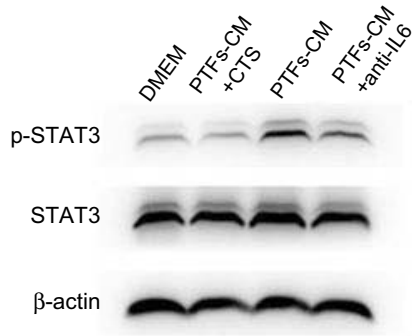

D

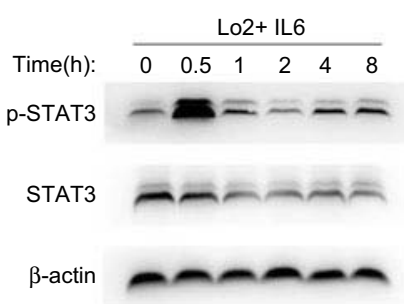

$\mathbf{F}$
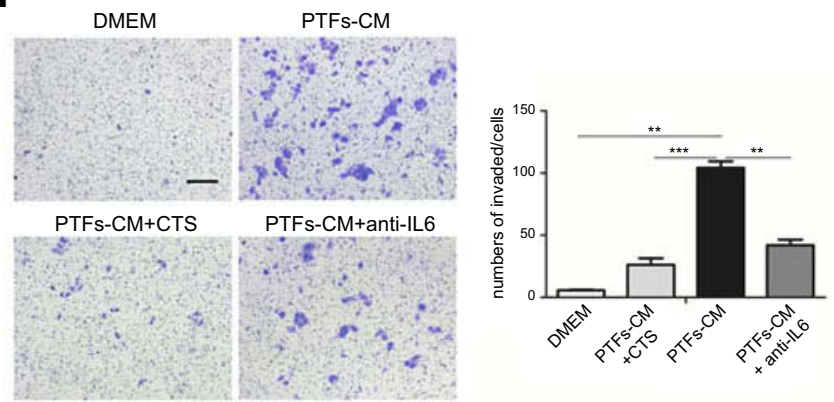

Figure 5 Effects of PTFs on the growth and migration of L-02 cells in vitro.

Notes: (A+B) DMEM and PTFs-CM cultured L-02 cells were assessed by colony forming assay $(\mathbf{A})$ and transwell assay (B) to examine cell proliferation and migration. (C) Western Blot analysis showed that PTFs induced the activation of STAT3 signaling in L-02 cells. Both CTS and IL-6 neutralizing antibody could reduce the effect. (D) L-02 cells were incubated with IL-6 (40 ng/ml). (E+F) L-02 cells cultured in PTFs-CM were treated with CTS or IL-6 neutralizing antibody for $24 \mathrm{~h}$ respectively. Then the cells were subjected to colonyforming assay $(\mathbf{E})$ and transwell assay $(\mathbf{F})$. Scale bar, $200 \mu \mathrm{m}$. Data are shown as mean \pm SD from at least three independent experiments $(* P<0.05$, $* * P<0.0 \mathrm{I}$, $* * * P<0.00 \mathrm{I})$.

Abbreviations: CM, conditioned medium; CTS, cryptotanshinone; PTFs, peri-tumor fibroblasts.

A
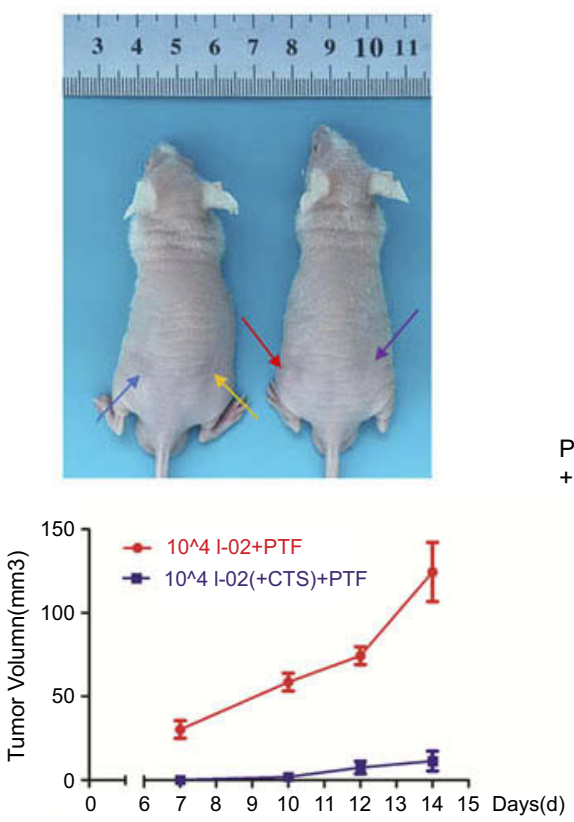

B

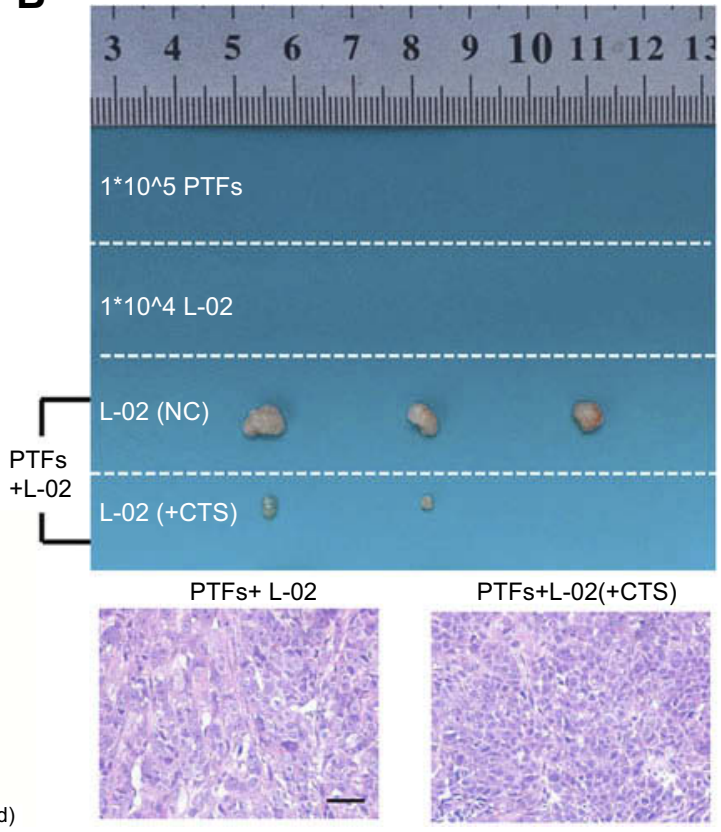

Figure 6 PTFs induced hepatocarcinogenesis through IL-6/P-STAT3 signaling in vivo.

Notes: (A) Non-tumor L-02 cells and PTFs were subcutaneously injected into BALB/c nude mice alone or together. Blue arrow means injecting PTFs; yellow arrow means $10^{4}$ L-02 cells; red arrow means $10^{4}$ L-02 cells injected with PTFs; purple arrow means $10^{4}$ CTS treated L-02 cells injected with PTFs. (B) Tumors formed two weeks after inoculation in each group are shown. Tumor formation was confirmed malignant by H\&E staining. Scale bar, $200 \mu \mathrm{m}$. $\mathrm{n}=3$.

Abbreviations: CTS, cryptotanshinone; PTFs, peri-tumor fibroblasts. 


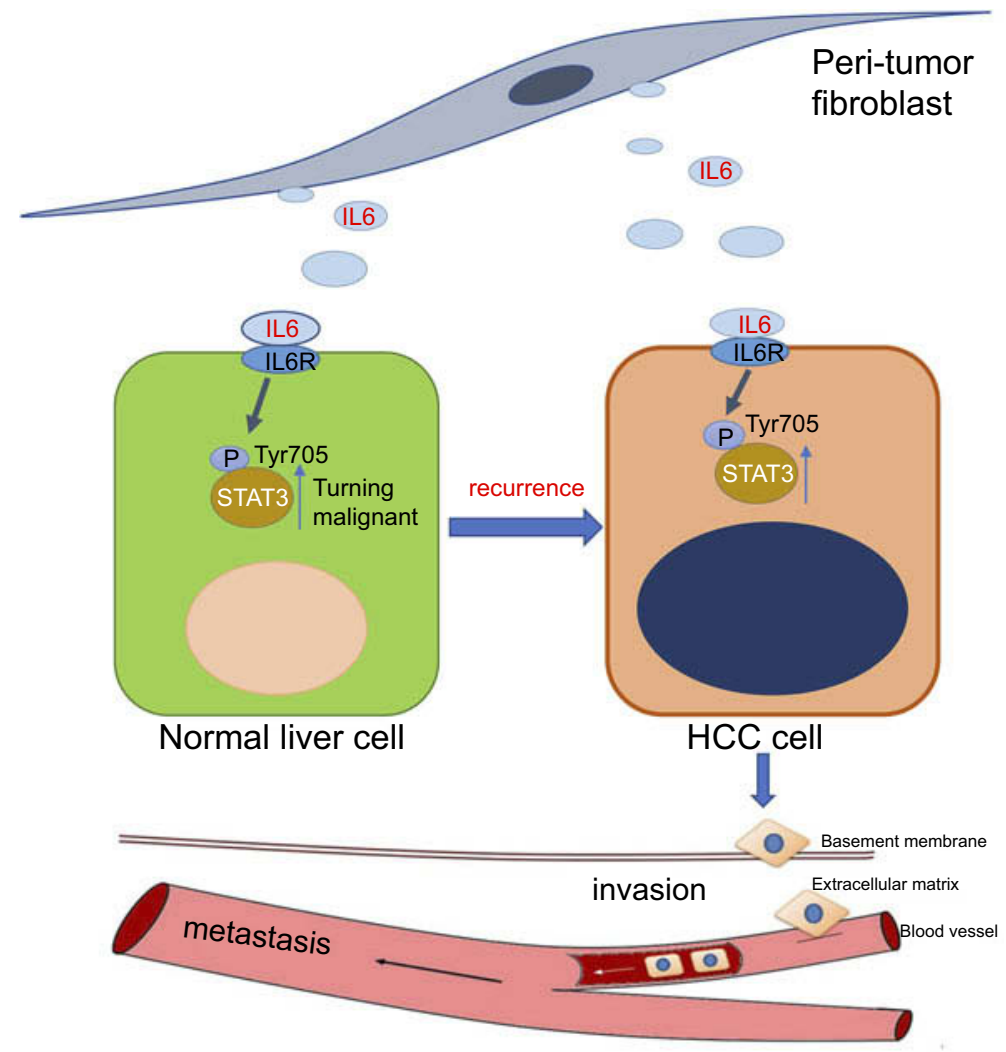

Figure 7 Hypothesis: after surgery, PTFs can stimulate surrounding hepatocytes to transform into a malignant phenotype, leading to hepatocarcinogenesis and tumor recurrence. Then PTFs significantly promote the progression and metastasis of HCC. IL-6/STAT3 signaling pathway plays a critical role in both stages. Abbreviations: HCC, hepatocellular carcinoma; PTFs, peri-tumor fibroblasts.

IL-22 also accelerate HCC development through activating STAT3 signaling. ${ }^{34,35}$ However, our data from the RayBio Human Cytokine Antibody Array and Elisa assays demonstrated that IL-6 was the major component secreted from PTFs that promoted STAT3 phosphorylation. Furthermore, the effect was largely abolished by the IL-6-neutralizing antibody.

Most HCC cases developed from fibrotic or cirrhotic livers, which are abundant in myofibroblasts derived from quiescent fibroblasts and hepatic stellate cells. ${ }^{36,37}$ Activated fibroblasts are characterized by certain mesenchymal markers, including $\alpha$-SMA and FAP. ${ }^{37}$ Our results were consistent with previous findings that CAFs express higher levels of $\alpha$-SMA and FAP compared to NFs or PTFs. However, a specific marker for PTFs should be defined if PTFs are to be considered when surgery margin reports are negative for HCC cells. Therefore, therapeutic strategies should be more selective and efficient in locating and removing PTFs from the livers of patients with HCC.

\section{Conclusion}

Our study demonstrates that PTFs stimulate the malignant transformation of non-tumor liver cells and promote hepatocarcinogenesis and tumor metastasis via paracrine IL-6-mediated STAT3 Tyr705 phosphorylation. Thus, we postulate that PTFs play a critical role in the postoperative recurrence and development of tumors (Figure 7). Our study suggests that PTFs should be considered as targets for therapeutic strategies made during the surgical resection and further management of HCC.

\section{Acknowledgment}

This work was supported by grants from the National Natural Science Foundation of China (81172063 and 81372352).

\section{Disclosure}

All authors report no conflicts of interest in this work. 


\section{References}

1. Bray F, Ferlay J, Soerjomataram I, Siegel RL, Torre LA, Jemal A. Global cancer statistics 2018: GLOBOCAN estimates of incidence and mortality worldwide for 36 cancers in 185 countries. CA Cancer J Clin. 2018;68(6):394-424.

2. Shim JH, Jun MJ, Han S, et al. Prognostic nomograms for prediction of recurrence and survival after curative liver resection for hepatocellular carcinoma. Ann Surg. 2015;261(5):939-946. doi:10.1097/ SLA.0000000000000747

3. Tabrizian P, Jibara G, Shrager B, Schwartz M, Roayaie S. Recurrence of hepatocellular cancer after resection: patterns, treatments, and prognosis. Ann Surg. 2015;261(5):947-955. doi:10.1097/SLA.000 0000000000710

4. Hanahan D, Coussens LM. Accessories to the crime: functions of cells recruited to the tumor microenvironment. Cancer Cell. 2012;21 (3):309-322. doi:10.1016/j.ccr.2012.02.022

5. Lau Eunice Yuen T, Lo J, Cheng Bowie Yik L, et al. Cancerassociated fibroblasts regulate tumor-initiating cell plasticity in hepatocellular carcinoma through c-Met/FRA1/HEY1 signaling. Cell Rep. 2016;15(6):1175-1189. doi:10.1016/j.celrep.2016.04.019

6. Liu J, Chen S, Wang W, et al. Cancer-associated fibroblasts promote hepatocellular carcinoma metastasis through chemokine-activated hedgehog and TGF-beta pathways. Cancer Lett. 2016;379(1):49-59.

7. Yang J, Lu Y, Lin YY, et al. Vascular mimicry formation is promoted by paracrine TGF-beta and SDF1 of cancer-associated fibroblasts and inhibited by miR-101 in hepatocellular carcinoma. Cancer Lett. 2016;383(1):18-27.

8. Jiang J, Ye F, Yang X, et al. Peri-tumor associated fibroblasts promote intrahepatic metastasis of hepatocellular carcinoma by recruiting cancer stem cells. Cancer Lett. 2017;404:19-28.

9. Xiong S, Wang R, Chen Q, et al. Cancer-associated fibroblasts promote stem cell-like properties of hepatocellular carcinoma cells through IL-6/STAT3/Notch signaling. Am J Cancer Res. 2018;8 (2):302-316.

10. Zheng X, Xu M, Yao B, Wang C, Jia Y, Liu Q. IL-6/STAT3 axis initiated CAFs via up-regulating TIMP-1 which was attenuated by acetylation of STAT3 induced by PCAF in HCC microenvironment. Cell Signal. 2016;28(9):1314-1324. doi:10.1016/j.cellsig.2016.06.009

11. Zhou M, Yang H, Learned RM, Tian H, Ling L. Non-cellautonomous activation of IL-6/STAT3 signaling mediates FGF19-driven hepatocarcinogenesis. Nat Commun. 2017;8:15433. doi: $10.1038 /$ ncomms 15433

12. Dauer P, Zhao X, Gupta VK, et al. Inactivation of cancer-associatedfibroblasts disrupts oncogenic signaling in pancreatic cancer cells and promotes its regression. Cancer Res. 2018;78(5):1321-1333. doi:10.1158/0008-5472.CAN-17-2320

13. Ishimoto T, Miyake K, Nandi T, et al. Activation of transforming growth factor beta 1 signaling in gastric cancer-associated fibroblasts increases their motility, via expression of rhomboid 5 homolog 2 , and ability to induce invasiveness of gastric cancer cells. Gastroenterology. 2017;153(1):191.e116-204.e116. doi:10.1053/j. gastro.2017.03.046

14. Wan S, Zhao E, Kryczek I, et al. Tumor-associated macrophages produce interleukin 6 and signal via STAT3 to promote expansion of human hepatocellular carcinoma stem cells. Gastroenterology. 2014;147(6):1393-1404. doi:10.1053/j.gastro.2014.08.039

15. Zhu XD, Zhang JB, Zhuang PY, et al. High expression of macrophage colony-stimulating factor in peritumoral liver tissue is associated with poor survival after curative resection of hepatocellular carcinoma. J Clin Oncol. 2008;26(16):2707-2716. doi:10.1200/ JCO.2007.15.6521
16. Ju MJ, Qiu SJ, Fan J, et al. Peritumoral activated hepatic stellate cells predict poor clinical outcome in hepatocellular carcinoma after curative resection. Am J Clin Pathol. 2009;131(4):498-510. doi:10.1309/ AJCP86PPBNGOHNNL

17. Su S, Chen J, Yao H, et al. CD10(+)GPR77(+) cancer-associated fibroblasts promote cancer formation and chemoresistance by sustaining cancer stemness. Cell. 2018;172(4):841-856. e816. doi:10.1016/j. cell.2018.01.009

18. Tsuyada A, Chow A, Wu J, et al. CCL2 mediates cross-talk between cancer cells and stromal fibroblasts that regulates breast cancer stem cells. Cancer Res. 2012;72(11):2768-2779. doi:10.1158/0008-5472. CAN-11-3567

19. Zhu L, Cheng X, Ding Y, et al. Bone marrow-derived myofibroblasts promote colon tumorigenesis through the IL-6/JAK2/STAT3 pathway. Cancer Lett. 2014;343(1):80-89. doi:10.1016/j. canlet.2013.09.017

20. Olumi AF, Grossfeld GD, Hayward SW, et al. Carcinoma-associated fibroblasts direct tumor progression of initiated human prostatic epithelium. Cancer Res. 1999;59(19):5002-5011.

21. Simon W. Hayward YW, Mei Cao, et al. Malignant transformation in a nontumorigenic human prostatic epithelial cell line. Cancer Res. 2001;61:8135-8142.

22. Zheng SG, Jia C-C, Wang T-T, et al. Cancer-associated fibroblasts from hepatocellular carcinoma promote malignant cell proliferation by HGF secretion. PLoS One. 2013;8(5):e63243.

23. Zhang Z, Li X, Sun W, et al. Loss of exosomal miR-320a from cancer-associated fibroblasts contributes to $\mathrm{HCC}$ proliferation and metastasis. Cancer Lett. 2017;397:33-42. doi:10.1016/j. canlet.2017.03.004

24. He G, Dhar D, Nakagawa H, et al. Identification of liver cancer progenitors whose malignant progression depends on autocrine IL-6 signaling. Cell. 2013;155(2):384-396. doi:10.1016/j.cell.2013.09.031

25 . Wang C, Sun H, Gao X, et al. Interleukin-6 enhances cancer stemness and promotes metastasis of hepatocellular carcinoma via up-regulating osteopontin expression. Am J Cancer Res. 2016;6 (9):1873-1889.

26. Karakasheva TA, Lin EW, Tang Q, et al. IL-6 mediates cross-talk between tumor cells and activated fibroblasts in the tumor microenvironment. Cancer Res. 2018;78(17):4957-4970. doi:10.1158/0008-5472.CAN-17-2268

27. Zhao M, Gao FH, Wang JY, et al. JAK2/STAT3 signaling pathway activation mediates tumor angiogenesis by upregulation of VEGF and bFGF in non-small-cell lung cancer. Lung Cancer. 2011;73 (3):366-374. doi:10.1016/j.lungcan.2011.01.002

28. Bohrer LR, Chuntova P, Bade LK, et al. Activation of the FGFR-STAT3 pathway in breast cancer cells induces a hyaluronan-rich microenvironment that licenses tumor formation. Cancer Res. 2014;74(1):374-386. doi:10.1158/0008-5472.CAN-132469

29. Yang Y, Zheng B, Han Q, et al. Targeting blockage of STAT3 inhibits Hepatitis B virus-related hepatocellular carcinoma. Cancer Biol Ther. 2016;17(4):449-456. doi:10.1080/ 15384047.2016.1156257

30. Peng D, Tanikawa T, Li W, et al. Myeloid-derived suppressor cells endow stem-like qualities to breast cancer cells through IL6/STAT3 and NO/NOTCH cross-talk signaling. Cancer Res. 2016;76 (11):3156-3165. doi:10.1158/0008-5472.CAN-15-2528

31. Zhu Q, Zhang X, Zhang L, et al. The IL-6-STAT3 axis mediates a reciprocal crosstalk between cancer-derived mesenchymal stem cells and neutrophils to synergistically prompt gastric cancer progression. Cell Death Dis. 2014;5:e1295. doi:10.1038/cddis.2014.263

32. Jang JW, Oh BS, Kwon JH, et al. Serum interleukin-6 and C-reactive protein as a prognostic indicator in hepatocellular carcinoma. Cytokine. 2012;60(3):686-693. doi:10.1016/j.cyto.2012.07.017 
33. Ohishi W, Cologne JB, Fujiwara S, et al. Serum interleukin-6 associated with hepatocellular carcinoma risk: a nested case-control study. Int $J$ Cancer. 2014;134(1):154-163. doi: $10.1002 /$ ijc. 28337

34. Zheng H, Yang Y, Han J, et al. TMED3 promotes hepatocellular carcinoma progression via IL-11/STAT3 signaling. Sci Rep. 2016;6:37070.
35. Jiang $\mathrm{R}$, Tan $\mathrm{Z}$, Deng $\mathrm{L}$, et al. Interleukin-22 promotes human hepatocellular carcinoma by activation of STAT3. Hepatology. 2011;54(3):900-909. doi:10.1002/hep.24486

36. Kalluri R, Zeisberg M. Fibroblasts in cancer. Nat Rev Cancer. 2006;6 (5):392-401. doi:10.1038/nrc1877

37. Kalluri R. The biology and function of fibroblasts in cancer. Nat Rev Cancer. 2016;16(9):582-598. doi:10.1038/nrc.2016.73

\section{Publish your work in this journal}

Cancer Management and Research is an international, peer-reviewed open access journal focusing on cancer research and the optimal use of preventative and integrated treatment interventions to achieve improved outcomes, enhanced survival and quality of life for the cancer patient
The manuscript management system is completely online and includes a very quick and fair peer-review system, which is all easy to use. Visit http://www.dovepress.com/testimonials.php to read real quotes from published authors. 